\title{
A proposed plan for personalized radiosurgery in patients with trigeminal neuralgia
}

\author{
Seyed H. Mousavi, MD, ${ }^{1}$ Ajay Niranjan, MD, ${ }^{1}$ Berkcan Akpinar, BA, ${ }^{3}$ Edward A. Monaco III, MD, PhD, ${ }^{1}$ \\ Jonathan Cohen, BS, ${ }^{3}$ Jagdish Bhatnagar, ScD, ${ }^{1,2}$ Yue-Fang Chang, PhD, ${ }^{1}$ Hideyuki Kano, MD, PhD, ${ }^{1}$ \\ Sakibul Huq, BS, ${ }^{1}$ John C. Flickinger, MD, ${ }^{2}$ and L. Dade Lunsford, MD ${ }^{1,2}$
}

\begin{abstract}
Departments of ${ }^{1}$ Neurosurgery and ${ }^{2}$ Radiation Oncology, University of Pittsburgh School of Medicine, University of Pittsburgh Medical Center; and ${ }^{3}$ University of Pittsburgh School of Medicine, Pittsburgh, Pennsylvania
\end{abstract}

\begin{abstract}
OBJECTIVE During the last 25 years, more than 100,000 patients worldwide with trigeminal neuralgia (TN) have undergone stereotactic radiosurgery (SRS) with a standard dose of radiation. However, the radiobiological effect of radiation is determined by the amount of energy delivered to the tissue (integral dose [ID] $=$ mean dose $\times$ target volume) and is directly associated with the nerve volume. Although the trigeminal nerve volume varies among patients with TN, the clinical impact of this variation in delivered energy is unknown. The objective of this study was to evaluate the effect of delivered ID on the outcome of TN radiosurgery.

METHODS The authors evaluated 155 patients with unilateral TN who had undergone SRS as their initial surgical management over a 13-year period. The authors measured the postganglionic ID within the SRS target and retrospectively stratified patients into 3 groups: low (<1.4 mJ), medium (1.4-2.7 mJ), and high (> $2.7 \mathrm{~mJ})$ ID. Clinical outcomes, which included pain status (scored using the Barrow Neurological Institute Pain Scale) and sensory dysfunction (scored using the Barrow Neurological Institute Numbness Scale), were evaluated at a median follow-up of 71 months.

RESULTS Patients who were treated with a medium ID had superior pain relief either with or without medications ( $p$ $=0.006$ ). In the medium ID group, the rates of complete pain relief without medications at 1, 3, and 6 years after SRS were $67 \%, 54 \%$, and $33 \%$, respectively, while the rates in the rest of the cohort were $55 \%, 36 \%$, and $19 \%$, respectively. Patients given a high ID had a higher rate of post-SRS trigeminal sensory deterioration $(p<0.0001)$. At 1, 3, and 6 years after SRS, the high ID group had an estimated rate for developing sensory dysfunction of $35 \%, 45 \%$, and $50 \%$, respectively, while the rates in patients receiving low and medium IDs were $3 \%, 4 \%$, and $9 \%$, respectively. The optimal clinical outcome (maximum pain relief and minimal trigeminal sensory dysfunction) was obtained in patients who had received a medium ID.
\end{abstract}

CONCLUSIONS With current dose selection methods, nerve volume affects long-term clinical outcomes in patients with TN who have undergone SRS. This study suggests that the prescribed SRS dose should be customized for each TN patient based on the nerve volume.

https://thejns.org/doi/abs/10.3171/2016.10.JNS16747

KEY WORDS trigeminal neuralgia; stereotactic radiosurgery; trigeminal nerve volume; integral dose; clinical outcome; pain

$\mathrm{S}$ TEREOTACTIC radiosurgery (SRS) delivers focused radiation from the decay of cobalt-60 in a single, minimally invasive procedure. The application of SRS is of interest in patients with medically refractory trigeminal neuralgia (TN) since it is considered the least invasive procedure for patients with associated comorbid conditions. ${ }^{11}$ The radiobiological effect of radiation is dependent on the amount of energy delivered (known as the integral dose
[ID] $)^{1}$ to the intended target. ID is the product of the mean dose and the target volume. ${ }^{10}$ Therefore, when the prescribed radiation target dose and treatment volume remain constant, the energy delivered to the tissue will increase proportionally to the volume of tissue within that target volume. For SRS in patients with TN, the treatment volume is usually an oblate spheroid (enclosing a segment of nerve and adjacent cerebrospinal fluid) and defined by the

ABBREVIATIONS BNI-NS = Barrow Neurological Institute Numbness Scale; BNI-PS = BNI Pain Scale; $\mathrm{Cl}=$ confidence interval; HR = hazard ratio; ID = integral dose; SRS = stereotactic radiosurgery; $\mathrm{TN}=$ trigeminal neuralgia.

SUBMITTED March 22, 2016. ACCEPTED October 4, 2016.

INCLUDE WHEN CITING Published online February 17, 2017; DOI: 10.3171/2016.10.JNS16747. 
4-mm collimator of the Leksell Gamma Knife. Although the trigeminal nerve volume varies among patients, the relationship between the ID delivered and clinical outcomes (defined as pain control and trigeminal sensory dysfunction) has not been studied. In this report, we evaluated the relationship between energy delivered and clinical outcomes in patients with TN who had undergone SRS as their initial surgical procedure over a 13-year period.

\section{Methods}

Patients

Between January 2002 and December 2014, 302 patients with unilateral medically refractory TN had undergone SRS as their initial surgical procedure. For this retrospective study, we excluded patients with atypical TN, patients who were unable to undergo MRI for target selection, patients with intracranial tumors that compressed the trigeminal nerve, patients with a follow-up less than 12 months, and patients with preexisting trigeminal sensory loss. The final study included 155 patients (mean age 71 years, 95\% CI 69-72 years), 57 males and 98 females. Patient follow-up information was collected from medi- cal records available in our system and supplemented by direct follow-up contact with the patient by a member of our team who was not involved in the patient's care. Pain outcomes were assessed using the Barrow Neurological Institute Pain Scale (BNI-PS), ${ }^{4}$ in which a score of I is defined as no pain on no medication, II as occasional pain but not requiring medication, III as no pain but on medication, IV as residual pain not adequately controlled with medication, and $\mathrm{V}$ as refractory pain without benefit from medication. Trigeminal sensory loss after SRS was evaluated using the BNI Numbness Scale (BNI-NS), ${ }^{4}$ in which a score of $\mathrm{I}$ is defined as no trigeminal sensory loss, II as mild but not bothersome trigeminal sensory loss, III as bothersome trigeminal sensory loss, and IV as very bothersome trigeminal sensory loss. The University of Pittsburgh approved our protocol for this retrospective study. Patient preradiosurgical demographic information is shown in Table 1.

\section{Procedures}

SRS had been performed as described previously. ${ }^{8}$ All procedures were performed with a model G Leksell ste-

TABLE 1. Preradiosurgery characteristics of typical patients with TN

\begin{tabular}{|c|c|c|c|c|c|c|c|}
\hline Characteristic & Low ID & Medium ID & High ID & $\begin{array}{l}\text { p Value, } \\
\text { Low vs } \\
\text { Medium }\end{array}$ & $\begin{array}{l}\text { p Value, } \\
\text { Medium } \\
\text { vs High }\end{array}$ & $\begin{array}{c}\text { p Value, } \\
\text { Low vs } \\
\text { High }\end{array}$ & Overall Cohort \\
\hline No. of patients & 33 & 79 & 43 & & & & 155 \\
\hline Age in yrs* & & & & 0.30 & 0.10 & 0.23 & \\
\hline Mean $(95 \% \mathrm{Cl})$ & $74(70-78)$ & $71(68-73)$ & 68 (64-71) & & & & $71(69-72)$ \\
\hline Sex (no.)† & & & & 0.002 & 0.70 & 0.002 & \\
\hline Male & 4 & 33 & 20 & & & & 57 \\
\hline Female & 29 & 46 & 23 & & & & 98 \\
\hline Pain side (no.)† & & & & 0.53 & 0.65 & 0.85 & \\
\hline Rt & 21 & 44 & 25 & & & & 90 \\
\hline Lt & 12 & 35 & 18 & & & & 65 \\
\hline Prior pain duration in mos* & & & & 0.11 & 0.75 & 0.14 & \\
\hline Mean $(95 \% \mathrm{Cl})$ & $55(38-72)$ & $76(62-89)$ & $80(57-103)$ & & & & $73(62-82)$ \\
\hline SRS target vol $\left(\mathrm{mm}^{3}\right)^{*}$ & & & & $<0.0001$ & $<0.0001$ & $<0.0001$ & \\
\hline Mean $(95 \% \mathrm{Cl})$ & $14.7(13.2-16.3)$ & $29.8(28.5-31.0)$ & $46.8(45.0-48.6)$ & & & & $31.3(29.3-33.2)$ \\
\hline SRS min dose on target vol (Gy)* & & & & 0.94 & 0.10 & 0.31 & \\
\hline Mean $(95 \% \mathrm{Cl})$ & $38.5(37.5-39.6)$ & $38.0(37.6-38.4)$ & $37.6(37.0-38.3)$ & & & & $38.0(37.7-38.3)$ \\
\hline SRS max dose on target vol (Gy)* & & & & 0.11 & 0.33 & 0.14 & \\
\hline Mean $(95 \% \mathrm{Cl})$ & $80.4(79.4-81.4)$ & $81.1(80.7-81.5)$ & $81.8(81.0-82.6)$ & & & & $81.1(80.8-81.4)$ \\
\hline SRS mean dose on target vol (Gy)* & & & & 0.24 & $<0.0001$ & 0.004 & \\
\hline Mean $(95 \% \mathrm{Cl})$ & $68.7(67.2-70.2)$ & $67.3(66.9-67.8)$ & $66.0(65.3-66.7)$ & & & & $67.2(66.8-67.7)$ \\
\hline SRS margin dose $(\mathrm{Gy})^{*}$ & & & & 0.74 & 0.11 & 0.32 & \\
\hline Mean $(95 \% \mathrm{Cl})$ & $40.5(40.1-40.8)$ & $40.4(40.2-40.6)$ & $40.8(40.4-41.2)$ & & & & $40.5(40.3-40.7)$ \\
\hline SRS max dose to brainstem (Gy)* & & & & 0.24 & 0.20 & 0.047 & \\
\hline Mean $(95 \% \mathrm{Cl})$ & $29.0(25.2-32.9)$ & $25.3(23.1-27.4)$ & $24.0(20.4-27.7)$ & & & & $25.7(24.1-27.4)$ \\
\hline SRS ID $(m J)^{*}$ & & & & $<0.0001$ & $<0.0001$ & $<0.0001$ & \\
\hline Mean $(95 \% \mathrm{Cl})$ & $1.01(0.91-1.12)$ & $2.00(1.92-2.08)$ & $3.11(2.99-3.22)$ & & & & $2.10(1.97-2.23)$ \\
\hline
\end{tabular}

Boldface type indicates statistical significance.

* Wilcoxon rank-sum test.

$\dagger$ Fisher exact test. 
reotactic frame (Elekta Instruments) and either the C, 4C, or Perfexion Gamma Knife. All patients underwent axial gadolinium-enhanced MRI using a $512 \times 256$ matrix with 1-mm slice thickness and whole-head, 3-mm, axial T2weighted imaging. Most patients also underwent axial 3D fast spin echo, 1-mm slice thickness T2-weighted MRI. Acquired images were exported to Leksell Gamma Knife computers, and a dose plan was created using the Leksell Gamma Plan. A 4-mm collimator was used to deliver the target dose (median dose was 80 Gy at the $100 \%$ isodose) for radiosurgical planning. The nerve volume inside the $50 \%$ isodose volume of the 4-mm isocenter was contoured retrospectively using stereotactic T1-weighted MRI studies obtained at the time of SRS. Accordingly, the minimum dose, maximum dose, and ID (mean dose $\times$ tissue volume) inside the $50 \%$ isodose line were calculated using dose-volume histograms as shown in Fig. 1. The mean nerve volume covered by the $50 \%$ isodose line was 31.3 $\mathrm{mm}^{3}$ (95\% CI 29.3-33.2 $\mathrm{mm}^{3}$ ), while the median was 31.6 $\mathrm{mm}^{3}$. Patients were further divided into 3 groups based on the ID inside the $50 \%$ isodose line that they had received: low $(\mathrm{n}=33$, ID $<1.4 \mathrm{~mJ})$, medium $(\mathrm{n}=79$, ID $1.4-2.7$ $\mathrm{mJ})$, and high $(\mathrm{n}=43, \mathrm{ID}>2.7 \mathrm{~mJ})$ ID groups. The nerve volume inside the $50 \%$ isodose line was used to calculate the ID (energy received by the target). The correlation between nerve volume and ID is shown as a scatterplot in Fig. 1. The ID was then correlated with pain outcomes and trigeminal nerve sensory dysfunction.

\section{Nerve Correlation Studies}

A subset of patients' nerves $(n=68)$, selected based on imaging availability, were further analyzed to determine the validity of using the $50 \%$ isodose nerve volume as a surrogate for the total cisternal nerve volume. Using the cisternal component of the treated nerve as outlined from the brainstem root entry point to the opening of Meckel's cave as our boundaries, we determined the total cisternal nerve volume and the respective ID delivered. Further, we measured the length of the cisternal component of each nerve. With these additional parameters, we performed a series of correlational analyses to assess the relationship between which specific anatomical parameters (50\% isodose line volume vs cisternal nerve volume vs cisternal nerve length) correlated best with ID delivered (to the 50\% isodose tissue vs to the cisternal nerve tissue).

\section{Statistical Analysis}

Any statistical comparison was considered significant if the $\mathrm{p}$ value was less than 0.05 . Patient pain relief (preservation of BNI-PS Scores I-III) and trigeminal sensory dysfunction (BNI-NS score $>$ I) outcome analyses were performed using the Kaplan-Meier approach. All confidence intervals (CIs) were calculated using the Greenwood method and quoted as $95 \%$ limits for each estimate referenced in the results and/or tables. Comparisons between groups (low, medium, and high ID groups) were performed using the Mantel-Cox log-rank test for Kaplan-Meier analysis. Continuous variables (patient age, SRS dose parameters, follow-up duration, duration of pre-SRS symptoms, and nerve volume) were compared using the Wilcoxon ranksum test. Comparison of patient sex and pain side (right vs left) within each group was done using the Fisher exact test. Cox proportional hazards regression models were used to elucidate potential contributors to the development of BNI-NS scores $>$ I and to the post-SRS deterioration of BNI-PS Scores I and I-III. To assess the development

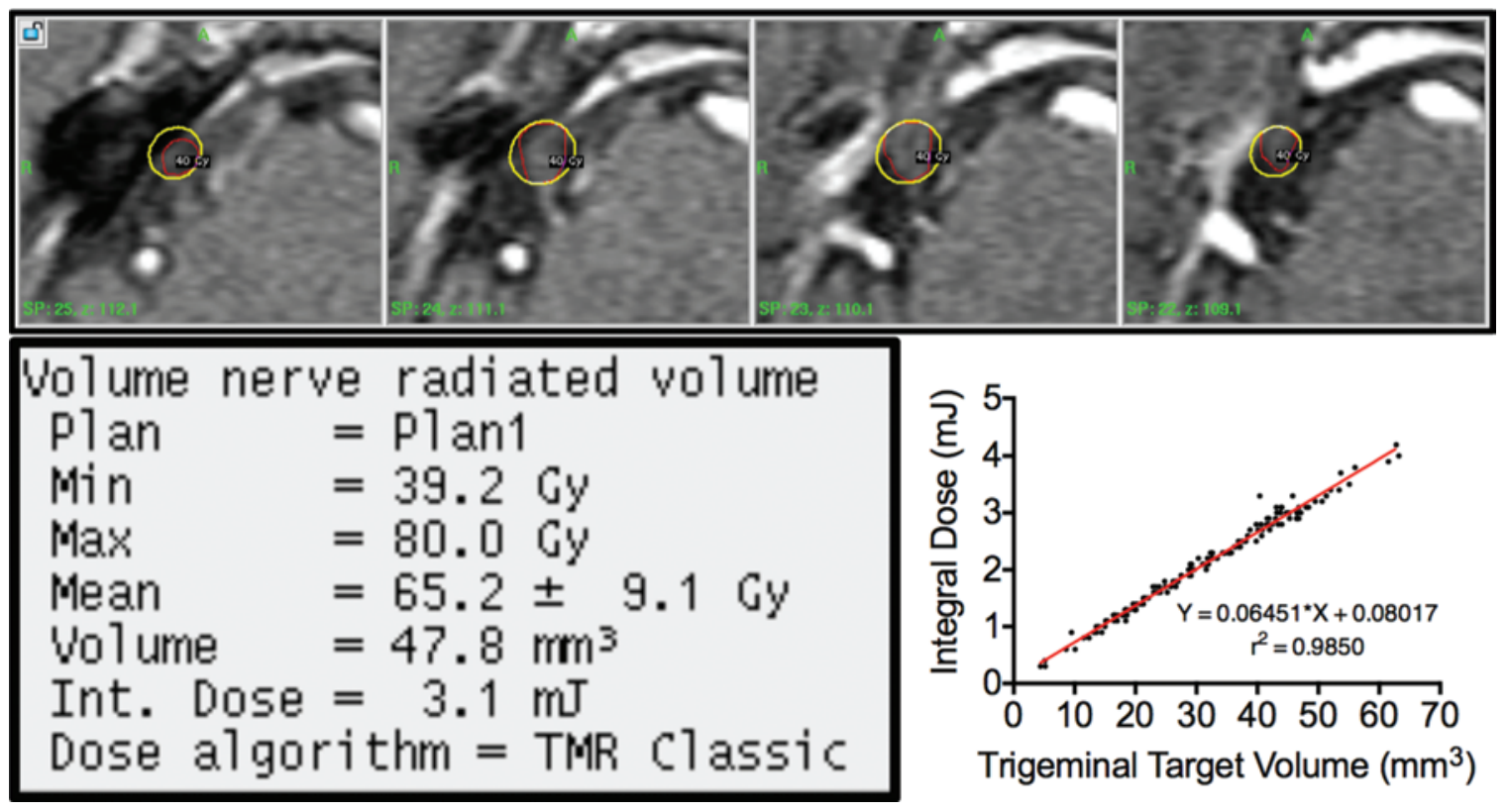

FIG. 1. Radiosurgical planning, volume, and ID correlation. Yellow circles depict the $50 \%$ isodose line (40 Gy). The area within the red lines is the trigeminal nerve volume inside the $50 \%$ isodose line. Target ID is calculated using mean dose and nerve volume (inside the red line). Trigeminal nerve target volume within the $50 \%$ isodose line was plotted against ID delivered to the trigeminal nerve within the $50 \%$ isodose line. A linear regression analysis was performed on this scatterplot (trend line: ID $=0.06451 \times$ target volume +0.08017 , Pearson $\left.r^{2}=0.985\right)$. Int. = integral. Figure is available in color online only. 
of BNI-NS scores > I, a Cox proportional hazards regression analysis with a stepwise selection procedure was performed to identify significant covariates using the following variables: age (continuous), sex (male vs female), duration of preradiosurgical pain (continuous), pain side (right vs left), and SRS dose parameters (mean dose in 50\% isodose line, minimum dose in $50 \%$ isodose line, maximum dose in $50 \%$ isodose line, margin dose, and maximum dose received by brainstem; all continuous). A non-stepwise Cox proportional hazards regression analysis was then performed to examine the relationship between nerve ID and the development of BNI-NS scores > I, while adjusting for those significant covariates identified by the stepwise procedure. Nerve ID was categorized into 3 groups (low, medium, and high), and the medium group was used as the referent group. To assess the risk of BNI-PS Scores I and IIII deterioration, a forward stepwise Cox proportional hazards regression analysis was performed using the following variables: age (continuous), sex (male vs female), duration of preradiosurgical pain ( $\leq 3$ vs $>3$ years), pain side (right vs left), development of post-SRS trigeminal sensory dysfunction (presence vs absence), and SRS dose parameters (mean dose in 50\% isodose line, minimum dose in 50\% isodose line, and maximum dose in $50 \%$ isodose line, margin dose, and maximum dose received by brainstem; all continuous). A non-stepwise Cox proportional hazards regression analysis was then performed with the variables deemed significant from the aforementioned forward stepwise analyses alongside patient nerve ID using the medium nerve ID as a reference. All hazard ratios (HRs) are reported with $95 \%$ CIs. The relationship between trigeminal nerve volume and ID delivered to the target tissue within the $50 \%$ isodose line was demonstrated using a scatterplot of patient nerve volume versus ID; the Pearson correlation coefficient $\left(r^{2}\right)$ was quoted for degree of curve linearity. Correlational studies between ID, nerve volume, and nerve length were performed using Pearson and Spearman correlation coefficients.

\section{Results}

To assess factors associated with superior pain relief, we performed an initial stepwise forward Cox proportional hazards regression analysis and identified earlier SRS ( $\leq 3$ years from diagnosis) as the only predictive factor ( $p$ $<0.0001$ ). Using a non-stepwise Cox proportional hazards regression, we found that earlier treatment $(p<0.0001)$ was a positive predictor of pain relief, while low ID ( $\mathrm{p}=$ $0.011)$ and high ID $(p=0.005)$ were negative predictors of pain relief (Table 2 and Fig. 2A). After SRS, patients who had received a medium ID experienced superior pain relief either with or without medications ( $\mathrm{p}=0.006$; Fig. 2A-D). At follow-ups of 1, 3, and 6 years after SRS, the medium ID group had the highest rate of complete pain relief without medications: $67 \%$ (95\% CI 57\%-77\%), 54\% (95\% CI 43\%-65\%), and 33\% (95\% CI 21\%-44\%), respectively. The remainder of the cohort had estimated rates of complete pain relief without medication of $55 \%$ (95\% CI $44 \%-66 \%), 36 \%(95 \%$ CI $25 \%-47 \%)$, and $19 \%$ (95\% CI 9\%-29\%) at the 1-, 3-, and 6-year follow-ups, respectively. Details of the pain outcomes are outlined in Table 3.
A

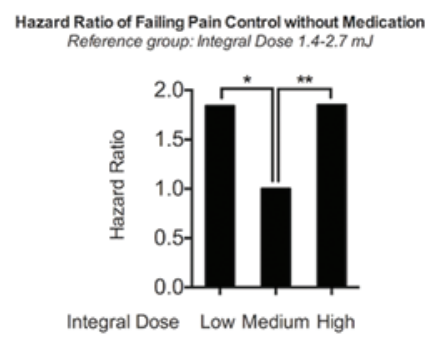

C

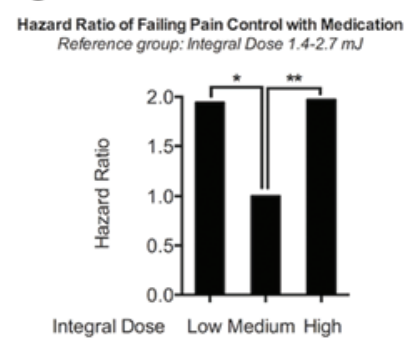

Integral Dose Low Medium High

E

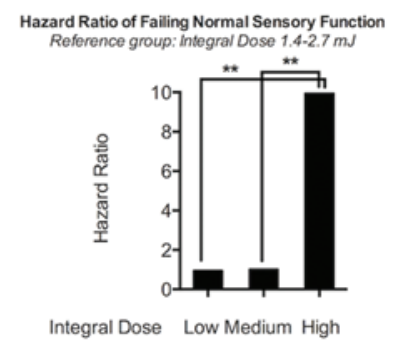

B

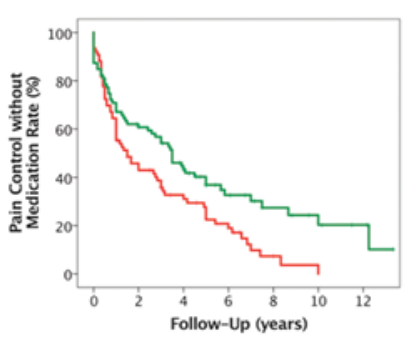

D

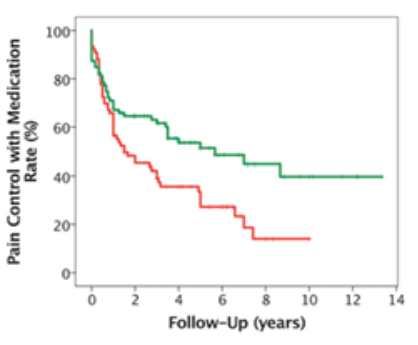

$\mathbf{F}$

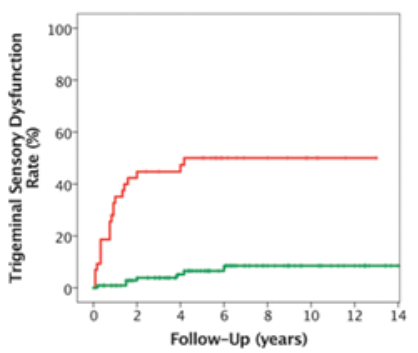

FIG. 2. Association between ID and clinical outcome. A: Cox proportional HRs for the 3 ID groups (low, medium, high) were plotted using the medium ID (1.4-2.7 mJ) as a reference group that was set to an HR of 1.00 for complete pain control without medications. B: Kaplan-Meier analysis of post-SRS rates of complete pain control without medications for the medium ID group (green) versus the rest of the population (red) $(p=0.006$, Mantel-Cox log-rank test). C: Cox proportional HRs for the 3 ID groups were plotted using the medium ID as a reference group that was set to an HR of 1.00 for complete pain control with medications. D: Kaplan-Meier analysis of post-SRS rates of complete pain control with medications for the medium ID group (green) versus the rest of the population (red) ( $p=0.006$, Mantel-Cox log-rank test). E: Cox proportional HRs for the 3 ID groups were plotted using the medium ID as a reference group that was set to an HR of 1.00 for normal sensory function deterioration risk. F: Kaplan-Meier analysis of post-SRS rates of normal sensory function deterioration risk for the high ID group (red) versus the rest of the population (green) $\left(p<0.0001\right.$, Mantel-Cox log-rank test). ${ }^{*} p$ $<0.05,{ }^{* *} p<0.01$. Figure is available in color online only.

To identify factors associated with trigeminal sensory dysfunction, we performed an initial stepwise forward Cox proportional hazards regression analysis and found that increased ID $(p=0.002)$ was predictive of trigeminal sensory dysfunction. However, in a non-stepwise Cox proportional hazards regression analysis, high ID $(\mathrm{p}<0.0001)$ was the only predictor of trigeminal sensory loss development (Table 2 and Fig. 2E). Patients with a high ID (> $2.7 \mathrm{~mJ}$ ) had a higher rate of post-SRS trigeminal sensory deterioration ( $\mathrm{p}<0.0001$; Fig. $2 \mathrm{~F}$ ) than patients in the low and medium ID groups. At 1, 3, and 6 years after SRS, the 
TABLE 2. Cox proportional hazards regression analyses

\begin{tabular}{|c|c|c|c|c|}
\hline \multirow[b]{2}{*}{ Variable } & \multicolumn{2}{|c|}{ Forward Stepwise Analysis } & \multicolumn{2}{|c|}{ Non-Stepwise Analysis } \\
\hline & $\mathrm{HR}(95 \% \mathrm{Cl}) \dagger$ & $p$ Value & $\mathrm{HR}(95 \% \mathrm{Cl}) \dagger$ & $p$ Value \\
\hline \multicolumn{5}{|l|}{ BNI-PS Score I deterioration risk } \\
\hline Patient age $\ddagger$ & - & 0.83 & - & - \\
\hline Sex (male vs female) & - & 0.14 & - & - \\
\hline Min dose on target volf & - & 0.58 & - & - \\
\hline Max dose on target volf & - & 0.53 & - & - \\
\hline Mean dose on target volf & - & 0.64 & - & - \\
\hline Margin dose $\ddagger$ & - & 0.25 & - & - \\
\hline Pain duration ( $\leq 3$ vs $>3$ yrs) & $0.31(0.20-0.49)$ & $<0.0001$ & $0.31(0.21-0.46)$ & $<0.0001$ \\
\hline Pain side (rt vs It) & - & 0.63 & - & - \\
\hline Max dose brainstem $\ddagger$ & - & 0.75 & - & - \\
\hline Post-SRS numbness (presence vs absence) & - & 0.94 & - & - \\
\hline Low target ID & - & - & $1.84(1.15-2.93)$ & 0.011 \\
\hline Medium target ID & - & - & * & * \\
\hline High target ID & - & - & $1.85(1.21-2.84)$ & 0.005 \\
\hline \multicolumn{5}{|l|}{ Trigeminal sensory dysfunction risk } \\
\hline Patient age & - & 0.46 & - & - \\
\hline Sex (male vs female) & - & 0.10 & - & - \\
\hline Min target dose & - & 0.84 & - & - \\
\hline Max target dose & - & 0.56 & - & - \\
\hline Mean target dosef & $0.85(0.74-0.99)$ & 0.034 & - & 0.63 \\
\hline Margin dose & $1.70(1.23-2.37)$ & 0.002 & - & 0.47 \\
\hline Duration of pain $\ddagger$ & - & 0.058 & - & - \\
\hline Pain side (rt vs It) & - & 0.96 & - & - \\
\hline Max dose brainstem $\ddagger$ & - & 0.18 & - & - \\
\hline Low target ID & - & - & - & 0.8 \\
\hline Medium target ID & - & - & $*$ & * \\
\hline High target ID & - & - & $9.95(3.50-28.28)$ & $<0.0001$ \\
\hline
\end{tabular}

TABLE 3. Pain relief outcomes: Kaplan-Meier analysis

\begin{tabular}{cccc}
\hline & \multicolumn{2}{c}{$\%(95 \% \mathrm{Cl})$} & $\mathrm{p}$ \\
\cline { 2 - 3 } Characteristic & Medium ID & Rest of Cohort & Value \\
\hline $\begin{array}{c}\text { BNI-PS Score I pres- } \\
\text { ervation rate* }\end{array}$ & & & 0.006 \\
\hline 1 yr & $67 \%(57 \%-77 \%)$ & $55 \%(44 \%-66 \%)$ & \\
\hline 3 yrs & $54 \%(43 \%-65 \%)$ & $36 \%(25 \%-47 \%)$ & \\
\hline 6 yrs & $33 \%(21 \%-44 \%)$ & $19 \%(9 \%-29 \%)$ & \\
\hline $\begin{array}{c}\text { BNI-PS Score I-III } \\
\text { preservation rate* }\end{array}$ & & & 0.006 \\
\hline 1 yr & $67 \%(57 \%-77 \%)$ & $56 \%(45 \%-68 \%)$ & \\
\hline 3 yrs & $62 \%(51 \%-72 \%)$ & $39 \%(28 \%-50 \%)$ & \\
\hline 6 yrs & $49 \%(36 \%-61 \%)$ & $23 \%(11 \%-35 \%)$ & \\
\hline
\end{tabular}

Boldface type indicates statistical significance.

${ }^{*}$ Mantel-Cox log-rank test. high ID group had an estimated rate for developing sensory dysfunction of $35 \%$ (95\% CI 21\%-49\%), $45 \%$ (95\% CI 30\%-60\%), and 50\% (95\% CI 35\%-65\%), respectively. Development of trigeminal sensory dysfunction at 1, 3, and 6 years post-SRS in patients who had received a low or medium ID was $3 \%$ (95\% CI 0\%-6\%), $4 \%(95 \% \mathrm{CI}$ $0 \%-8 \%$ ), and $9 \%$ (95\% CI 2\%-15\%), respectively (Table 4). Two patients within the high ID group developed deafferentation pain after SRS.

A schematic of the relationship between ID and clinical outcome is depicted in Fig. 3 left. Using a quadratic Cox regression model with target ID as a continuous variable, we determined that an ID between 1.4 and $2.7 \mathrm{~mJ}$ was the optimum range for best pain relief with the least risk of sensory loss. To optimize the mean dose selection for best outcomes for any nerve volume, we generated a hypothetical ID curve to optimize outcomes (Fig. 3 right).

Finally, the degree of correlation between the 50\% isodose line and its respective ID was the strongest of all correlation analyses performed (Pearson $\mathrm{r}^{2}=0.994$, $\mathrm{p}<$ 
TABLE 4. Trigeminal sensory dysfunction outcomes: KaplanMeier analysis

\begin{tabular}{cccc}
\hline \multirow{2}{*}{ Variable } & High ID & Rest of Cohort & $\begin{array}{c}p \\
\text { Value }\end{array}$ \\
\cline { 2 - 3 } & & & $<0.0001$ \\
\hline $\begin{array}{c}\text { Trigeminal sensory } \\
\text { dysfunction de- } \\
\text { velopment rate* }\end{array}$ & & \\
\hline 1 yr & $35 \%(21 \%-49 \%)$ & $3 \%(0 \%-6 \%)$ & \\
\hline 3 yrs & $45 \%(30 \%-60 \%)$ & $4 \%(0 \%-8 \%)$ & \\
\hline 6 yrs & $50 \%(35 \%-65 \%)$ & $9 \%(2 \%-15 \%)$ & \\
\hline
\end{tabular}

Boldface type indicates statistical significance.

* Mantel-Cox log-rank test.

0.0009). The degree of correlation between the trigeminal nerve cisternal volume and its respective ID demonstrated a strong correlation as well (Pearson $\mathrm{r}^{2}=0.919$, $\mathrm{p}$ $<0.0009$ ). The total nerve length demonstrated the least correlational relationship with ID delivered to the $50 \%$ isodose tissue (Pearson $\mathrm{r}^{2}=0.441, \mathrm{p}<0.0009$ ) as well as with the total cisternal nerve tissue (Pearson $\mathrm{r}^{2}=0.475$, $\mathrm{p}$ $<0.0009)$. Figure 4 and Table 5 display all correlations that were assessed regarding ID and nerve metric parameters.

\section{Discussion}

More than 60 years ago, Mayneord introduced the concept of ID, ${ }^{10}$ which was defined as the total energy absorbed by the target volume of tissue (tissue volume $\times$ mean dose it receives). By definition, if the radiation dose remains constant, ID increases as the radiated target volume increases. More importantly, the desired effect and the risks of radiation depend on ID. ${ }^{2}$ SRS is a widely used method to precisely deliver focused radiation and has been applied

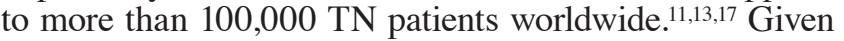
its minimally invasive nature, it is now considered to be a primary option for management of TN in patients who are poor surgical candidates. ${ }^{11}$ SRS exerts its radiobiological
TABLE 5. Correlations between total nerve and $50 \%$ isodose line component in terms of ID and tissue volume in a random subset of 68 patients

\begin{tabular}{lcccc}
\hline $\begin{array}{c}\text { Relationship } \\
\text { Compared* }\end{array}$ & $\begin{array}{c}\text { Pearson } \\
\text { Coefficient }\end{array}$ & $\begin{array}{c}p \\
\text { Value }\end{array}$ & $\begin{array}{c}\text { Spearman } \\
\text { Coefficient }\end{array}$ & $\begin{array}{c}p \\
\text { Value }\end{array}$ \\
\hline $\begin{array}{c}50 \% \text { isodose line nerve } \\
\text { vol to } 50 \% \text { isodose } \\
\text { line ID }\end{array}$ & 0.994 & $<0.0009$ & 0.992 & $<0.0009$ \\
\hline $\begin{array}{c}\text { Total nerve vol to total } \\
\text { nerve ID }\end{array}$ & 0.919 & $<0.0009$ & 0.925 & $<0.0009$ \\
\hline $\begin{array}{c}\text { Total nerve ID to 50\% } \\
\text { isodose line ID }\end{array}$ & 0.832 & $<0.0009$ & 0.846 & $<0.0009$ \\
\hline $\begin{array}{c}50 \% \text { isodose line nerve } \\
\text { vol to total nerve vol }\end{array}$ & 0.788 & $<0.0009$ & 0.834 & $<0.0009$ \\
\hline $\begin{array}{c}\text { Total nerve vol to 50\% } \\
\text { isodose line ID }\end{array}$ & 0.783 & $<0.0009$ & 0.829 & $<0.0009$ \\
\hline $\begin{array}{c}50 \% \text { isodose line nerve } \\
\text { vol to total nerve ID }\end{array}$ & 0.834 & $<0.0009$ & 0.848 & $<0.0009$ \\
\hline $\begin{array}{c}\text { Total nerve length to } \\
50 \% \text { isodose line ID }\end{array}$ & 0.441 & $<0.0009$ & 0.449 & $<0.0009$ \\
\hline $\begin{array}{c}\text { Total nerve length to } \\
\text { total nerve ID }\end{array}$ & 0.475 & $<0.0009$ & 0.488 & $<0.0009$ \\
\hline
\end{tabular}

Boldface type indicates statistical significance.

* Cisternal nerve segment.

effect on the trigeminal nerve by axonal and myelin sheath damage and destruction of ionic channels, resulting in a blockade of electrical conduction. ${ }^{6,14}$ The radiation dose that leads to sufficient pain control is considered to be the best therapeutic dose. However, when the radiobiological damage results in major axonal damage, it is likely that patients will also develop trigeminal neuropathy.

In the current study, we found that the delivered ID varies substantially (varying by as much as a factor of 10) depending on the volume of the trigeminal nerve that is irradiated. We demonstrated that the target ID plays a significant role in long-term clinical outcomes after SRS. Our results show that by evaluating the trigeminal nerve target
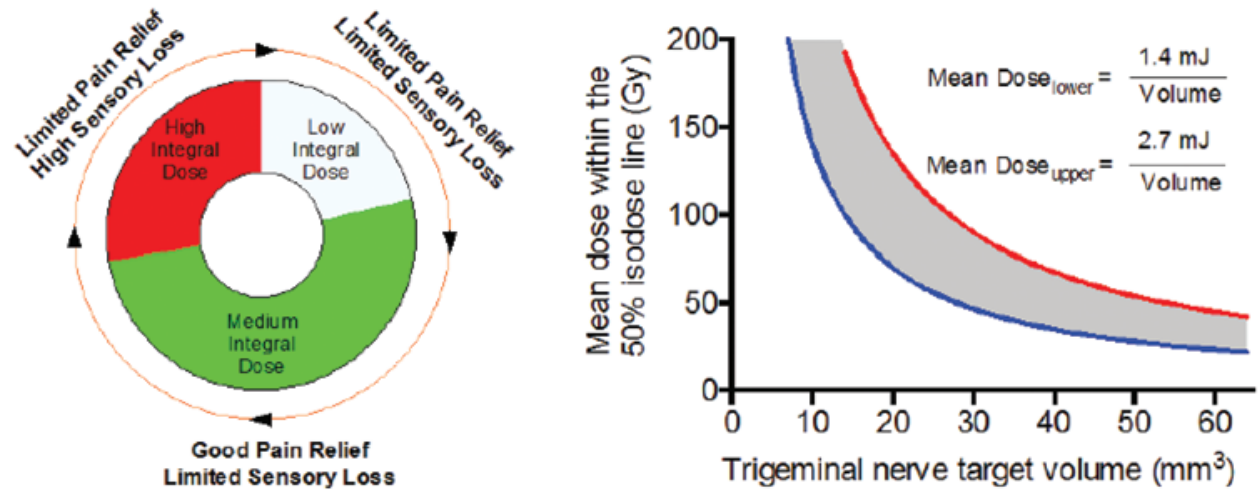

FIG. 3. Left: Relationship between ID and clinical outcome. The white area reflects 33 patients who had received an ID $<1.4 \mathrm{~mJ}$, the green area reflects 79 patients who had received an ID between 1.4 and $2.7 \mathrm{~mJ}$, and the red area reflects 43 patients who had received an ID $>2.7 \mathrm{~mJ}$. The relative outcome of each group is depicted adjacent to each area. The best outcome (good pain relief and limited sensory loss) was noted for the medium ID group. Right: Hypothetical mean dose range, depending on the trigeminal nerve target volume, for best clinical outcomes based on optimum ID (lower limit: blue; upper limit: red). Figure is available in color online only. 
A

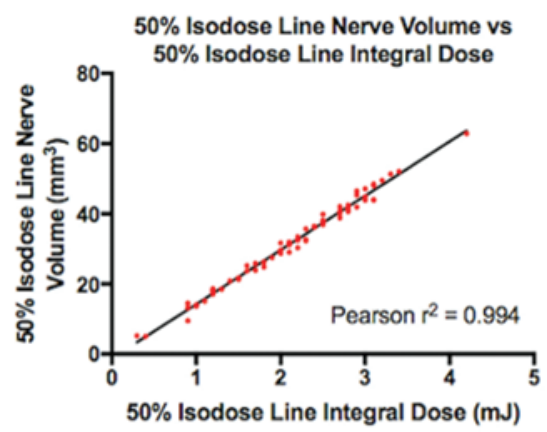

C

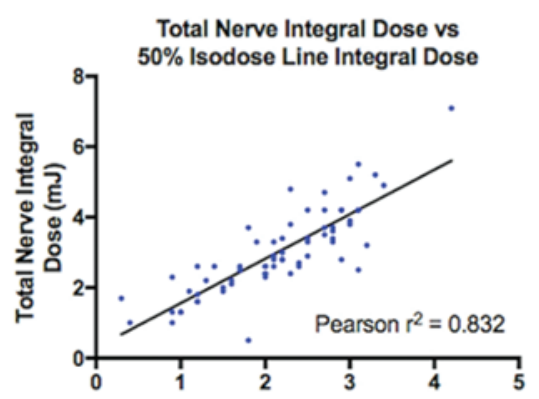

E

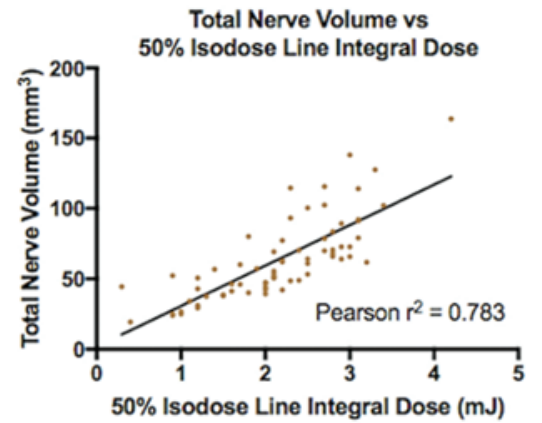

G

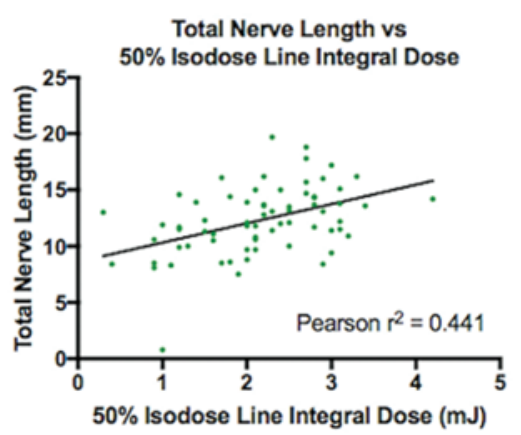

B

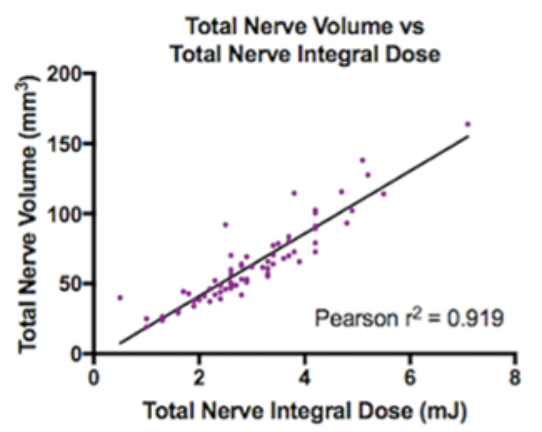

D

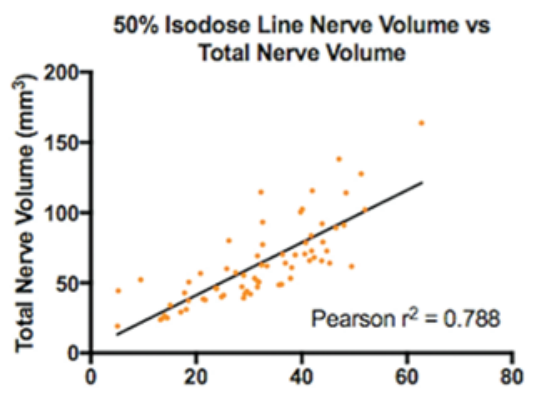

$50 \%$ Isodose Line Nerve Volume $\left(\mathrm{mm}^{3}\right)$
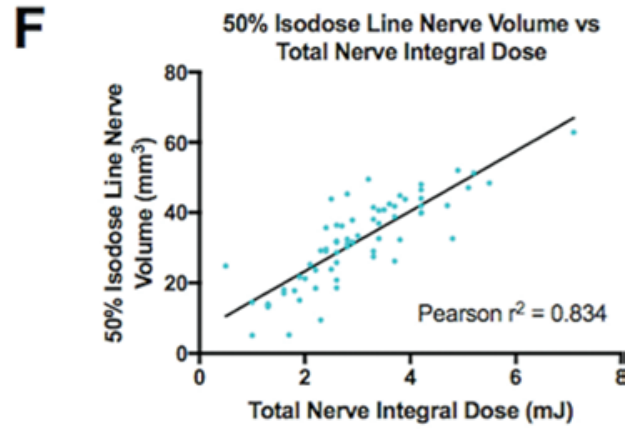

Total Nerve Length vs

Total Nerve Integral Dose

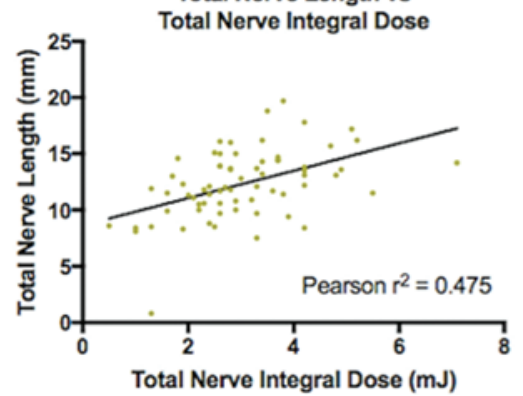

FIG. 4. A subset $(n=68)$ of patient nerves was analyzed for various correlations among nerve volumetric and ID-related parameters (A, C, E, G: $50 \%$ isodose line nerve volume vs $50 \%$ isodose line ID; B, D, F, H: total nerve volume vs total nerve ID). Patients in this cohort received a mean maximum dose of $81.4 \mathrm{~Gy}(95 \% \mathrm{Cl} 80.9-81.9 \mathrm{~Gy}$, range $80-85 \mathrm{~Gy})$. The strength of correlations was demonstrated using the Pearson correlation coefficient, and all relationships were significant $(p<0.0009)$. Figure is available in color online only.

volume and adjusting the delivered dose, pain relief can be achieved with a low risk of additional trigeminal sensory loss. In fact, in this study, trigeminal sensory loss was not associated with pain relief after SRS, whereas controversial results (sensory loss was associated with better pain relief) are reported in prior studies. ${ }^{5,7,9,12,16}$ This divergence of opinion may be related, in part, to the heterogeneity of the study cohorts. For example, the majority of patients enrolled in prior studies underwent SRS as their second or third surgical procedure. In contrast, the present study evaluated only patients without prior surgery. All patients had received a standard maximum target dose of 80-85 Gy to the trigeminal target in a single outpatient procedure. We found that patients with a larger postganglionic 
nerve volume (and who received a higher ID) had a higher risk of developing trigeminal neuropathy without improving pain relief. This finding is supported by our previously published, prospective, randomized, double-blinded study, in which we found that a larger volume of radiated postganglionic nerve (and therefore higher ID) led to increased sensory loss but no improvement in pain outcome. ${ }^{3}$ Moreover, in a recent study by Senova et al., patients with vestibular schwannoma who received a higher ID delivered to the trigeminal nerve were more likely to develop trigeminal neuropathy. ${ }^{15}$ To maximize pain control while minimizing the risk of sensory loss, data in the current study suggest that the prescribed dose should be carefully tailored to the volume of the nerve in individual patients to optimize the ID delivered. Lastly, the current study demonstrates the utility of using the $50 \%$ isodose line ID as a surrogate for the total ID delivered to the total cisternal nerve volume, thereby supporting the use of the 50\% isodose line ID as a potential prognostic index for post-SRS pain relief and trigeminal sensory dysfunction. In the future, a prospective trial that optimizes ID may provide a higher level of scientific evidence. Such a trial is currently under consideration by the International Gamma Knife Research Foundation.

\section{Conclusions}

In order to maximize pain control while minimizing the risk of sensory loss, the prescribed dose should be carefully tailored to the volume of the nerve enclosed in the $50 \%$ isodose volume, leading to a delivered ID of $1.4-2.7 \mathrm{~mJ}$.

\section{References}

1. Aoyama H, Westerly DC, Mackie TR, Olivera GH, Bentzen SM, Patel RR, et al: Integral radiation dose to normal structures with conformal external beam radiation. Int J Radiat Oncol Biol Phys 64:962-967, 2006

2. D'Arienzo M, Masciullo SG, de Sanctis V, Osti MF, Chiacchiararelli L, Enrici RM: Integral dose and radiationinduced secondary malignancies: comparison between stereotactic body radiation therapy and three-dimensional conformal radiotherapy. Int J Environ Res Public Health 9:4223-4240, 2012

3. Flickinger JC, Pollock BE, Kondziolka D, Phuong LK, Foote RL, Stafford SL, et al: Does increased nerve length within the treatment volume improve trigeminal neuralgia radiosurgery? A prospective double-blind, randomized study. Int J Radiat Oncol Biol Phys 51:449-454, 2001

4. Gellner V, Kurschel S, Kreil W, Holl EM, Ofner-Kopeinig P, Unger F: Recurrent trigeminal neuralgia: long term outcome of repeat Gamma Knife radiosurgery. J Neurol Neurosurg Psychiatry 79:1405-1407, 2008

5. Kano H, Kondziolka D, Yang HC, Zorro O, Lobato-Polo J, Flannery TJ, et al: Outcome predictors after Gamma Knife radiosurgery for recurrent trigeminal neuralgia. Neurosurgery 67:1637-1645, 2010

6. Kondziolka D, Lacomis D, Niranjan A, Mori Y, Maesawa S, Fellows W, et al: Histological effects of trigeminal nerve radiosurgery in a primate model: implications for trigeminal neuralgia radiosurgery. Neurosurgery 46:971-977, 2000
7. Kondziolka D, Perez B, Flickinger JC, Habeck M, Lunsford LD: Gamma Knife radiosurgery for trigeminal neuralgia: results and expectations. Arch Neurol 55:1524-1529, 1998

8. Kondziolka D, Zorro O, Lobato-Polo J, Kano H, Flannery TJ, Flickinger JC, et al: Gamma Knife stereotactic radiosurgery for idiopathic trigeminal neuralgia. J Neurosurg 112:758765,2010

9. Marshall K, Chan MD, McCoy TP, Aubuchon AC, Bourland JD, McMullen KP, et al: Predictive variables for the successful treatment of trigeminal neuralgia with Gamma Knife radiosurgery. Neurosurgery 70:566-573, 2012

10. Mayneord WV: The measurement of radiations for medical purposes. Proc Phys Soc 54:405, 1942

11. Mousavi SH, Niranjan A, Huang MJ, Laghari FJ, Shin SS, Mindlin JL, et al: Early radiosurgery provides superior pain relief for trigeminal neuralgia patients. Neurology 85:21592165,2015

12. Pollock BE, Phuong LK, Foote RL, Stafford SL, Gorman DA: High-dose trigeminal neuralgia radiosurgery associated with increased risk of trigeminal nerve dysfunction. Neurosurgery 49:58-64, 2001

13. Régis J, Tuleasca C, Resseguier N, Carron R, Donnet A, Gaudart J, et al: Long-term safety and efficacy of Gamma Knife surgery in classical trigeminal neuralgia: a 497-patient historical cohort study. J Neurosurg 124:1079-1087, 2016

14. Schwarz W, Fox JM: Effects of monochromatic X-radiation on the membrane of nodes of Ranvier under voltage and current clamp conditions. Experientia 35:1200-1201, 1979

15. Senova S, Aggad M, Golmard JL, Hasboun D, Lamproglou I, Jenny C, et al: Predictors of trigeminal neuropathy after radiosurgery for vestibular schwannomas. Int J Radiat Oncol Biol Phys 95:721-728, 2016

16. Sheehan J, Pan HC, Stroila M, Steiner L: Gamma Knife surgery for trigeminal neuralgia: outcomes and prognostic factors. J Neurosurg 102:434-441, 2005

17. Takanashi M, Fukuoka S, Ozaki Y, Satou K, Oikawa M, Nakamura H: [Gamma Knife radiosurgery for trigeminal neuralgia: analysis of a multi institutional study.] No Shinkei Geka 41:1065-1074, 2013 (Jpn)

\section{Disclosures}

Dr. Lunsford is a stockholder in Elekta AB.

\section{Author Contributions}

Conception and design: Mousavi. Acquisition of data: Mousavi, Akpinar, Cohen. Analysis and interpretation of data: Mousavi, Akpinar. Drafting the article: Mousavi, Akpinar. Critically revising the article: Lunsford, Mousavi, Niranjan, Akpinar, Monaco, Bhatnagar, Flickinger. Reviewed submitted version of manuscript: Lunsford, Mousavi, Niranjan, Akpinar, Monaco, Cohen, Bhatnagar, Chang, Huq, Flickinger. Approved the final version of the manuscript on behalf of all authors: Lunsford. Statistical analysis: Mousavi, Akpinar, Chang, Kano, Flickinger. Administrative/ technical/material support: Huq. Study supervision: Lunsford, Niranjan.

\section{Correspondence}

L. Dade Lunsford, Department of Neurosurgery, F158 UPMC Presbyterian, 200 Lothrop St., Pittsburgh, PA 15213. email: lunsfordld@upmc.edu. 